\title{
Forgetting in Managing Rules and Ontologies*
}

\author{
Thomas Eiter, Giovambattista Ianni, \\ Roman Schindlauer, Hans Tompits \\ Institut für Informationssysteme 184/3, \\ Technische Universität Wien, \\ Favoritenstrasse 9-11, \\ A-1040 Vienna, Austria \\ \{eiter, ianni, roman, tompits\}@kr.tuwien.ac.at
}

\author{
Kewen Wang \\ School of Information and \\ Communication Technology, \\ Griffith University, \\ Brisbane, QLD 4111, Australia \\ k.wang@cit.gu.edu.au
}

\begin{abstract}
The language of HEX-programs under the answer-set semantics is designed for interoperating with heterogeneous sources via external atoms and for meta-reasoning via higher-order literals in the context of the Semantic Web. As an important technique in managing knowledge bases, the notion of forgetting has received increasing interest in the knowledge-representation area. In this paper, we introduce a semantics-based theory of forgetting for HEX-programs and, in turn, for a class of OWL/RDF ontologies which allows to fully employ semantic information in managing ontologies like editing, merging, aligning, and redundancy removal.
\end{abstract}

\section{Introduction}

An ontology is a formal representation of concepts and relationships between them, making global interoperability possible. Managing ontologies is a central task for many Semantic-Web applications. However, it is often acknowledged that the Ontology Layer of the Semantic Web [1] is insufficient in its reasoning abilities. In particular, more and more ontologies are available on the Web and they are often very large in size and heterogeneous in location.

This phenomenon brings up a good deal of challenges to researchers in the Semantic Web. For example, when an ontology design is involved, we have to consider some issues like how to tailor an ontology or how to merge ontologies. Recently, these and related issues of managing ontologies have received considerable interests $[12,17,18$, $9,10,7]$. Related issues include ontology editing, ontology

* This work was partially supported by the Austrian Science Fund (FWF) under grant P17212-N04, as well as by the European Commission through the IST Networks of Excellence REWERSE (IST-2003-506779). segmentation, ontology merging, ontology aligning, ontology reusing, ontology update, and ontology redundancy removal. To some extent, all of these issues can be reduced to the problem of extracting relevant segments out of large ontologies for the purpose of effective management of ontologies so that the tractability for both humans and computers is enhanced. Such segments are not mere fragments of ontologies, but stand alone as ontologies in their own right. The intuition here is similar to views in databases: an existing ontology is tailored to a smaller ontology so that an optimal ontology is produced for specific applications. Although this problem has been identified and a number of approaches are proposed, like, e.g., [8, 20], a general framework for tailoring ontologies in a purely semantic way is still missing.

On the other hand, the notion of forgetting $[4,15,14]$ is a promising technique for adequately handling a range of classical tasks such as query answering, planning, decisionmaking, reasoning about actions, or knowledge update and revision. The idea of forgetting consists, informally, in the intelligent and "painless" removal of information from a given knowledge base. In other words, one may select some literals, predicates, or concepts, for being discarded (or forgotten) in a given knowledge base. However, the information selected for elimination is usually logically connected with other portions of the same knowledge base. It is thus important to preserve, to the best extent, soundness and completeness of the information entailed after removal.

While a literature on forgetting in logic programming exists (see, e.g., [22, 4]), and although forgetting takes relevance also in ontology-description formalism such as OWL, an explicit notion of forgetting has not been given yet for this class of languages. In this respect, the relationship between a notion of forgetting in ontologies and of forgetting in rule-based formalisms has not satisfactorily been investigated yet, and is thus matter of new research.

The problem of forgetting in ontologies can indeed be 
solved by exploiting the connection between ontologydescription formalisms and logic programming. That is, given a sound notion of forgetting for logic programming, a knowledge base $L$, formulated under a generic semantics (e.g. RDFS, OWL, etc.) can be transposed to an equivalent logic program $P_{L}$, formulated under a different (and usually, nonmonotonic) semantics. Then, logic programming forgetting techniques are applied to $P_{L}$ and a modified program, forget $\left(P_{L}, l\right)$, is obtained and translated back to a knowledge base $L^{\prime}$, where $l$ is the information to be discarded, which can be either a propositional atom, a concept, or a predicate.

Nonetheless, in order to fulfill the above approach, several issues, some of which already tackled in the literature, have to be solved and accommodated:

- A systematic way for translating $L$ to $P_{L}$ must be given. Attempts in this direction are several: for instance, Grosof et al. [11] translate a fragment of OWLDL to Horn logic, whereas Swift [21] and Motik, Volz, and Maedche [16] port significant fragments of description logics to positive disjunctive logic programs.

- The pre-existing forgetting semantics [22,4] mainly concentrates on discarding propositional information from ground programs. However, often $P_{L}$ might be a non-ground program and $l$ a non-propositional value (such as a predicate whose entire extension must be discarded). Also, many ontology description languages (such as RDF and RDFS) include the possibility of exchanging the notion of class with the notion of individual, in order to enable meta-reasoning. In such a setting, $P_{L}$ is better mapped to a higher-order logic program.

- Also it is unclear in which cases forget $\left(P_{L}, l\right)$ can be mapped back to a valid knowledge base $L^{\prime}$.

In the present paper, we aim at answering some of the questions above.

The logic programming language of choice is HEX, as defined in previous work [3]. This is a rule-based, fully declarative formalism which allows both for higher-order atoms and external atoms, under a well-defined generalization of the answer-set semantics [6].

Intuitively, a higher-order atom allows to quantify values over predicate names and to freely exchange predicate symbols with constant symbols, like in the rule $C(X) \leftarrow$ subClassOf $(D, C), D(X)$. An external atom facilitates the assignment of a truth value of an atom through an external source of computation. For instance, the rule $t($ Sub, Pred,$O b j) \leftarrow \& r d f[u r i](S u b$, Pred,$O b j)$ computes the predicate $t$ taking values from the predicate $\& r d f$. The latter extracts RDF statements from the set of URIs specified by the extension of the predicate uri; this task is delegated to an external computational source (e.g., an external deduction system, an execution library, etc.). External atoms allow for a bidirectional flow of information to and from external sources of computation such as description-logic reasoners. By means of HEX-programs, powerful meta-reasoning becomes available in a decidable setting, e.g., not only for Semantic-Web applications, but also for meta-interpretation techniques in answer-set programming (ASP) itself, or for defining policy languages.

The contributions in this paper can be summarized as follows:

1. We introduce the notion of semantic forgetting for HEX-programs. Forgetting in logic programs has been previously considered by Eiter and Wang [4], who defined forgetting of a given literal $l$ in the context of propositional disjunctive logic programs. This notion is extended in order to deal with external and higherorder atoms, as well as with positive non-ground programs.

2. We develop an algorithm for forgetting which is useful in the setting of ontology management. The basic idea of this algorithm is that certain rules that are locally redundant may become relevant afterwards and thus they are kept in the program.

3. We show how semantic forgetting of ontologies can be performed using an equivalent logic program, whose modified versions (after forgetting) are translated back to ontologies. In particular, that fragment of OWLDL is taken into account which can be translated to description-logic programs [11]. The approach can be currently generalized to all those ontology languages for which a sound and complete mapping to positive logic programs is known.

Our approach is illustrated on some example application. For this, we use an ontology "Person-Relationship" in the paper, which can be scaled as large as one wishes.

The rest of the paper is organized as follows. Section 2 briefly recalls syntax and semantics of HEX-programs. Section 3 introduces the notion of semantic forgetting for HEXprograms and a novel algorithm for computing forgetting. As well, forgetting for non-ground positive programs is defined. Section 4 , then, discusses a method for forgetting OWL/RDF-ontologies in terms of a transformation technique. Finally, Section 5 wraps up the paper with some concluding remarks.

\section{HEX-Programs}

\subsection{Syntax}

HEX programs are built on mutually disjoint sets $\mathcal{C}, \mathcal{X}$, 
and $\mathcal{G}$ of constant names, variable names, and external predicate names, respectively. Unless stated otherwise, elements from $\mathcal{X}$ (resp., $\mathcal{C}$ ) are written with first letter in upper case (resp., lower case), and elements from $\mathcal{G}$ are prefixed with " \&." Constant names serve both as individual and predicate names. Importantly, $\mathcal{C}$ may be infinite.

Elements from $\mathcal{C} \cup \mathcal{X}$ are called terms. A higherorder atom (or atom) is a tuple $\left(Y_{0}, Y_{1}, \ldots, Y_{n}\right)$, where $Y_{0}, \ldots, Y_{n}$ are terms and $n \geq 0$ is its arity. Intuitively, $Y_{0}$ is the predicate name; we thus also use the familiar notation $Y_{0}\left(Y_{1}, \ldots, Y_{n}\right)$. The atom is ordinary, if $Y_{0}$ is a constant. For example, $(x, r d f$ :type, $c)$ and node $(X)$ are ordinary atoms, while $D(a, b)$ is a higher-order atom. An external atom is of the form

$$
\& g\left[Y_{1}, \ldots, Y_{n}\right]\left(X_{1}, \ldots, X_{m}\right)
$$

where $Y_{1}, \ldots, Y_{n}$ and $X_{1}, \ldots, X_{m}$ are two lists of terms (called input list and output list, respectively), and $\& g$ is an external predicate name.

It is possible to specify molecules of atoms similar as in F-Logic [13]. For instance, gi ffather $\rightarrow X, Z \rightarrow i u]$ is a shortcut for the conjunction father $(g i, X), Z(g i, i u)$.

A HEX-program ${ }^{1}$ is a set of rules of the form

$$
\alpha \leftarrow \beta_{1}, \ldots, \beta_{n}, \text { not } \beta_{n+1}, \ldots, \text { not } \beta_{m},
$$

where $m \geq 0, \alpha$ is a higher-order atom, and $\beta_{1}, \ldots, \beta_{m}$ are either higher-order atoms or external atoms. The operator "not" is negation as failure (or default negation). For a rule $r$ as in (2), we define $h e a d(r)=\alpha$ and $\operatorname{body}(r)=$ $\operatorname{body}^{+}(r) \cup b o d y^{-}(r)$, where body ${ }^{+}(r)=\left\{\beta_{1}, \ldots, \beta_{n}\right\}$ and body ${ }^{-}(r)=\left\{\beta_{n+1}, \ldots, \beta_{m}\right\}$. If $r$ contains only ordinary atoms, then $r$ is ordinary. Furthermore, $r$ is quasi-negative if $n=0$. A HEX-program is quasi-negative if it contains only quasi-negative rules. An ordinary rule is positive iff $m=n$, i.e., if it contains no negation as failure. A program is positive iff all rules in it are positive.

We mention that higher-order features in logic programs have also been considered, e.g., by Chen, Kifer, and Warren [2] and Ross [19].

\subsection{Semantics}

The semantics of HEX-programs [3] is defined by generalizing the answer-set semantics [6]. The Herbrand base of a program $P$, denoted $H B_{P}$, is the set of all possible ground versions of atoms and external atoms occurring in $P$ obtained by replacing variables with constants from $\mathcal{C}$. The grounding of a rule $r, \operatorname{grnd}(r)$, is defined accordingly, and the grounding of program $P$ is given by

\footnotetext{
${ }^{1}$ In contrast to the original definition in [3], here we consider only HEXprograms without disjunctions in rule heads.
}

$\operatorname{grnd}(P)=\bigcup_{r \in P} \operatorname{grnd}(r)$. Unless specified otherwise, $\mathcal{C}$, $\mathcal{X}$, and $\mathcal{G}$ are implicitly given by $P$.

Any subset $I \subseteq H B_{P}$ containing only atoms is called an interpretation relative to $P$. We say that an interpretation $I$ is a model of an atom $a \in H B_{P}$ iff $a \in I$. Furthermore, $I$ is a model of a ground external atom $a=\& g\left[y_{1}, \ldots\right.$, $\left.y_{n}\right]\left(x_{1}, \ldots, x_{m}\right)$ iff

$$
f_{\& g}\left(I, y_{1}, \ldots, y_{n}, x_{1}, \ldots, x_{m}\right)=1,
$$

where $f_{\& g}$ is an $(n+m+1)$-ary Boolean function associated with $\& g$, called oracle function, assigning each element of $2^{H B_{P}} \times \mathcal{C}^{n+m}$ either 0 or 1 . We write $I \models a$ to express that $I$ is a model of $a$.

Let $r$ be a ground rule. We define (i) $I \models \operatorname{body}(r)$ iff $I \models a$ for all $a \in \operatorname{body} y^{+}(r)$ and $I \not \models a$ for all $a \in \operatorname{body}(r)$, and (ii) $I \models r$ iff $I \models h e a d(r)$ whenever $I \models \operatorname{bod} y(r)$. We say that $I$ is a model of a HEX-program $P$, denoted $I \models P$, iff $I \models r$ for all $r \in \operatorname{grnd}(P)$.

The Faber-Leone-Pfeifer reduct [5] (or FLP-reduct, for short) of $P$ with respect to $I \subseteq H B_{P}$, denoted $f P^{I}$, is the set of all $r \in \operatorname{grnd}(P)$ such that $I \models \operatorname{body}(r) . I \subseteq H B_{P}$ is an answer set of $P$ iff $I$ is a minimal model of $f P^{I}$. By $\mathrm{AS}(P)$ we denote the set of all answer sets of $P$.

A HEX-program is consistent if it has at least one answer set. We call two HEX-programs, $P$ and $Q$, equivalent, symbolically $P \equiv Q$, iff $\mathrm{AS}(P)=\operatorname{AS}(Q)$.

In practice, it is useful to differentiate between two kinds of input attributes for external atoms. For an external predicate $\& g$ (exploited, say, in an atom $\& g[p](X))$, a term appearing in an attribute position of type predicate (in this case, $p$ ) means that the outcomes of $f_{\& g}$ are dependent from the current interpretation $I$, for what the extension of the predicate named $p$ in $I$ is concerned. An input attribute of type constant does not imply a dependency of $f_{\& g}$ from some portion of $I$. An external predicate whose input attributes are all of type constant does not depend from the current interpretation.

Example 1 The external predicate \& $r d f$ introduced before is implemented with a single input argument of type predicate, because its associated function finds the RDF-URIs in the extension of the predicate $u r i$ :

$$
\begin{aligned}
\operatorname{tr}(S, P, O) & \leftarrow \& r d f[u r i](S, P, O), \\
\text { uri ("file://foaf.rdf" }) & \leftarrow .
\end{aligned}
$$

Should the input argument be of type constant, an equivalent program would be:

$$
\operatorname{tr}(S, P, O) \leftarrow \& r d f[" \text { file://foaf.rdf"] }(S, P, O)
$$

or

$$
\begin{aligned}
\operatorname{tr}(S, P, O) & \leftarrow \& r d f[X](S, P, O), \operatorname{uri}(X), \\
\text { uri("file://foaf.rdf") } & \leftarrow .
\end{aligned}
$$




\section{Forgetting in HEX-Programs}

As we have explained in Section 1, the technique of forgetting is useful in managing ontologies. So it is natural and interesting to generalize forgetting to HEX-programs. In fact, since HEX-programs have higher-order syntax but first-order semantics, it allows us to adapt the notion of forgetting to HEX-programs. In this section, we introduce the notion of forgetting for HEX-programs. The intuition behind the forgetting of an atom $l$ in a HEX-program is to obtain a HEX-program which is equivalent to the original HEX-program if we ignore the existence of $l$.

In the next subsection, we assume that HEX-programs are ground and consistent. When a HEX-program with variables is given, it is a shorthand for its ground version. As we will see in Section 4, forgetting in an RDF ontology is defined in terms of forgetting in the corresponding logic program, which is a non-ground positive program. So, in Subsection 3.2, forgetting in non-grounded positive programs is considered.

\subsection{Forgetting in Ground HEx-Programs}

We call a set $X^{\prime}$ an $l$-subset of a set $X$, denoted $X^{\prime} \subseteq_{l}$ $X$, if $X^{\prime} \backslash\{l\} \subseteq X \backslash\{l\}$. Similarly, a set $X^{\prime}$ is a strict $l$ subset of $X$, denoted $X^{\prime} \subset_{l} X$, if $X^{\prime} \backslash\{l\} \subset X \backslash\{l\}$. Two sets $X$ and $X^{\prime}$ of literals are l-equivalent, denoted $X \sim_{l}$ $X^{\prime}$, if $\left(X \backslash X^{\prime}\right) \cup\left(X^{\prime} \backslash X\right) \subseteq\{l\}$.

Definition 1 Let $P$ be a consistent HEX-program, let $l$ be a (ground) atom in $P$, and let $X$ be a set of atoms.

1. For a collection $\mathcal{S}$ of sets of atoms, $X \in \mathcal{S}$ is $l$-minimal in $\mathcal{S}$ if there is no $X^{\prime} \in \mathcal{S}$ such that $X^{\prime} \subset_{l} X$.

2. An answer set $X$ of a HEX-program $P$ is an $l$-answer set if $X$ is l-minimal in $\operatorname{AS}(P)$.

Example 2 Let $P=\{p \leftarrow$ not $q ; q \leftarrow$ not $p ; s \leftarrow$ $p ; s \leftarrow q\}$. It is easy to see that $P$ has two answer sets, viz. $X=\{p, s\}$ and $X^{\prime}=\{q, s\}$. Then, $X$ is a $p$-answer set but $X^{\prime}$ is not.

Having defined the notion of minimality about forgetting an atom, we are now in a position to define the result of forgetting about an atom in a HEX-program.

Definition 2 Let $P$ be a consistent HEX-program and let $l$ be a (ground) atom. A HEX-program $P^{\prime}$ is a result of forgetting about $l$ in $P$, if $P^{\prime}$ represents $l$-answer sets of $P$, i.e., such that the following conditions are satisfied:

1. At $\left(P^{\prime}\right) \subseteq A t(P)-\{l\}$, where, for any program $Q$, $A t(Q)$ denotes the set of atoms occurring in $Q$.
2. For any set $X^{\prime}$ of atoms with $l \notin X^{\prime}, X^{\prime}$ is an answer set of $P^{\prime}$ iff there is an l-answer set $X$ of $P$ such that $X^{\prime} \sim_{l} X$.

Note that the first condition implies that $l$ does not appear in $P^{\prime}$. We use forget $(P, l)$ to denote a possible result of forgetting about $l$ in $P$.

Since an atom that does not appear in the head of a rule in a HEX-program is automatically assumed to be false in the process of forgetting for ordinary programs, all external atoms would be removed from the program. For this reason, the native algorithm for forgetting [4] is not helpful for HEX-programs. Thus, we introduce a new algorithm, which is inspired by Algorithm 4 in the system LPForget. ${ }^{2}$

Preparatory for describing the algorithm, below we introduce some program transformations for HEX-programs, which are generalizations of respective ones for ordinary programs [4].

In the following, let $P$ and $P^{\prime}$ be HEX-programs.

Elimination of Tautologies: $P^{\prime}$ is obtained from $P$ by elimination of tautologies iff there is a rule $r$ in $P$ such that head $(r) \in \operatorname{body} y^{+}(r)$ and $P^{\prime}=P-\{r\}$.

Elimination of Head Redundancy: $P^{\prime}$ is obtained from $P$ by elimination of head redundancy iff there is a rule $r$ in $P$ such that head $(r) \in b o d y^{-}(r)$ and $P^{\prime}=$ $(P-\{r\}) \cup\{\leftarrow \operatorname{body}(r)\}$.

Positive Reduction: $P^{\prime}$ is obtained from $P$ by positive reduction iff there is a rule $r$ in $P$ such that $b o d y^{-}(r)$ contains some $c$ which does not occur in the head of any rule in $P$ and $P^{\prime}$ is obtained from $P$ by removing not $c$ from $r$.

Negative Reduction: $P^{\prime}$ is obtained from $P$ by negative reduction iff there are two rules $r$ and $r^{\prime}: b^{\prime} \leftarrow$ in $P$ such that $b^{\prime} \in \operatorname{bod} y^{-}(r)$ and $P^{\prime}=P-\{r\}$.

Elimination of Implications: Let $r$ and $r^{\prime}$ be two distinct rules in a logic program. We say that $r^{\prime}$ is an implication of $r$ if head $(r)=\operatorname{head}\left(r^{\prime}\right)$ and $\operatorname{body}(r) \subset$ $\operatorname{body}\left(r^{\prime}\right)$. Then, $P^{\prime}$ is obtained from $P$ by elimination of implications iff there are two distinct rules $r$ and $r^{\prime}$ of $P$ such that $r^{\prime}$ is an implication of $r$ and $P^{\prime}=P-\left\{r^{\prime}\right\}$.

Unfolding: For two rules $r$ and $r^{\prime}$ such that head $\left(r^{\prime}\right) \in$ body ${ }^{+}(r)$, the unfolding of $r$ with $r^{\prime}$, symbolically $\operatorname{unfold}\left(r, r^{\prime}\right)$, is the rule head $(r) \leftarrow \operatorname{body}(r)-$ $\left\{\right.$ head $\left.\left.\left(r^{\prime}\right)\right\}\right), \operatorname{body}\left(r^{\prime}\right)$. Then, $P^{\prime}$ is obtained from $P$ by unfolding if there is a rule $r$ such that

$$
\begin{aligned}
& P^{\prime}=(P-\{r\}) \cup\left\{\operatorname{unfold}\left(r, r^{\prime}\right) \mid r^{\prime} \in P,\right. \\
& \text { head } \left.\left(r^{\prime}\right) \in \operatorname{body}{ }^{+}(r)\right\} \text {. }
\end{aligned}
$$

\footnotetext{
${ }^{2}$ See http://www. cit.gu. edu. au/ kewen/LPForget/.
} 
Algorithm 1 (Computing a result of forgetting)

Input: HEX-program $P$ and an atom $l$ in $P$.

Output: Program forget $(P, l)$ as a result of forgetting $l$ from $P$.

Method:

Step 1. Positive Splitting: Initially take $Q$ as the set of all rules in which $l$ appears. For every rule $r$ in $P$ such that either head $(r)$ or some literal of body $(r)$ appears in $Q$, add $r$ to $Q$. Repeat this process until no new rule can be added. The resulting program is still denoted $Q$.

Step 2. Fully apply on $Q$ the program transformations $\mathcal{T}$ and then obtain a quasi-negative program $Q^{\prime}$. During this process, we keep record of the set $R U(Q, l)$ of all rules removed by unfolding but containing no appearance of $l$.

Step 3. Suppose that $Q^{\prime}$ has $n$ rules with head $l$ :

$$
r_{j}: l \leftarrow \text { not } l_{j 1}, \ldots, \text { not } l_{j m_{j}},
$$

where $n \geq 0, j=1, \ldots, n$ and $m_{j} \geq 0$ for all $j$.

If $n=0$, then let $Q^{\prime \prime}$ denote the program obtained from $Q^{\prime}$ by removing all appearances of not $l$.

If $n=1$ and $m_{1}=0$, then $l \leftarrow$ is the only rule in $Q^{\prime}$ having head $l$. In this case, remove every rule in $Q^{\prime}$ whose body contains not $l$. Let $Q^{\prime \prime}$ be the resulting program.

For $n \geq 1$ and $m_{1}>0$, let $D_{1}, \ldots, D_{s}$ be all possible conjunctions $\left(l_{1 k_{1}}, \cdots, l_{n k_{n}}\right)$, where $0 \leq k_{1} \leq m_{1}, \ldots$, $0 \leq k_{n} \leq m_{n}$. Replace each occurrence of not $l$ in $Q^{\prime}$ by all possible $D_{i}$. Let $Q^{\prime \prime}$ be the result.

Step 4. Output $Q^{\prime \prime} \cup R U(Q, l) \cup \bar{Q}$ as forget $(P, l)$, where $\bar{Q}=P \backslash Q$.

\section{Figure 1. Algorithm 1 for computing a result of forgetting.}

A special case of unfolding is when there is no rule $r^{\prime}$ such that $r^{\prime}$ is resolved with $r$. In this case, $P^{\prime}=$ $P-\{r\}$.

We use $\mathcal{T}$ to denote the set of program transformations introduced above.

Lemma 1 By using program transformations from $\mathcal{T}$, every HEX-program can be transformed into a quasi-negative program such that no atom appears in both head and body of a rule.

The algorithm for computing the result of forgetting, referred to as Algorithm 1, is depicted in Figure 1. This algorithm can be easily implemented using the system LPForget. Note that the current form of Algorithm 1 is incomplete with respect to the semantic forgetting for some special cases while it is intuitive and can be seen an ideal

\begin{tabular}{|c|c|}
\hline Male $\sqsubseteq$ Person & parentOf $($ Bob, Emma $)$ \\
\hline Female $\sqsubseteq$ Person & sameProject(Bob, Dennis) \\
\hline$\top \sqsubseteq \forall k$ nows $^{-}$.Person & spouseOf $\sqsubseteq$ knows \\
\hline$\top \sqsubseteq \forall k n o w s . P e r s o n$ & spouseOf $\equiv$ spouseOf ${ }^{-}$ \\
\hline friendOf $\sqsubseteq$ knows & works With $\equiv$ works With ${ }^{-}$ \\
\hline childOf $\sqsubseteq$ knows & works With $^{+} \sqsubseteq$ works With \\
\hline siblingOf $\equiv$ sibling $O f^{-}$ & worksWith $\sqsubseteq$ knows \\
\hline siblingOf ${ }^{+} \sqsubseteq$ siblingOf & parentOf $\equiv$ childOf ${ }^{-}$ \\
\hline siblingOf $\sqsubseteq$ knows & parentOf $\sqsubseteq$ ancestor $O f$ \\
\hline parentOf $($ Alice, Bob) & ancestorOf $\sqsubseteq$ knows \\
\hline parentOf (Alice, Carl) & $\begin{array}{l}\text { ancestorOf } f^{+} \sqsubseteq \text { ancestorOf } \\
\text { sameProject } \sqsubseteq \text { works With }\end{array}$ \\
\hline
\end{tabular}

Figure 2. Example ontology $L$.

approximation to the semantic forgetting. A complete algorithm is obtained by replacing Step 3 with Step 3 of Algorithm 2 given by Eiter and Wang [4].

For a consistent HEX-program $P$ and an atom $l$, some program $P^{\prime}$ as in Definition 2 always exists. However, different such programs $P^{\prime}$ might exist. It follows from the above definition that they are all equivalent under the answer-set semantics.

Proposition 1 Let $P$ be a HEX-program and $l$ an atom in $P$. If $P^{\prime}$ and $P^{\prime \prime}$ are two results of forgetting about $l$ in $P$, then $P^{\prime} \equiv P^{\prime \prime}$.

Example 3 Suppose that $L$ is a knowledge base on the Web consisting of various axioms about persons and their relationships. In particular, $L$ contains assertions depicted in Figure 2.

Let $P$ now be the following HEX-program, where $\& d l C$ and $\& d l R$ are external atoms that query the extensions of a specified concept resp. role from a single description logic ontology: ${ }^{3}$

$$
\begin{aligned}
\operatorname{sibling}(X, Y) \leftarrow & \& d l R[\operatorname{sibling} O f](X, Y) ; \\
\operatorname{sibling}(X, Y) \leftarrow & \& d l R[\operatorname{childOf}](X, Z), \\
& \& d l R[\operatorname{child} O f](Y, Z) ; \\
\operatorname{inEurope}(Y) \leftarrow & \operatorname{sibling}(\text { "Bob", } Y), \\
& \text { not inAmerica }(Y) ; \\
\operatorname{inAmerica}(Y) \leftarrow & \operatorname{sibling}(\text { "Bob", } Y), \\
& \text { not inEurope }(Y) .
\end{aligned}
$$

To apply forgetting, we first have to obtain the ground program $\operatorname{grnd}(P)$. In order to keep the example readable, we

\footnotetext{
${ }^{3}$ For the sake of readability, we use a simplified version of the actual and implemented dl-atoms for HEX-programs here.
} 
omit those ground rules whose bodies are not satisfied by $L$ :

$$
\begin{aligned}
& \text { sibling ("Bob", "Carl") } \leftarrow \& d l R[\text { childOf]("Bob", } \\
& \text { "Alice"), } \\
& \& \operatorname{dlR}[\text { childOf]( "Carl", } \\
& \text { "Alice"); } \\
& \text { sibling("Carl", "Bob") } \leftarrow \& d l R[\text { childOf }] \text { ("Carl", } \\
& \text { "Alice"), } \\
& \text { \&dlR }[\text { childOf]("Bob", } \\
& \text { "Alice"); } \\
& \text { inEurope("Carl") } \leftarrow \operatorname{sibling}(" B o b ", \text { "Carl"), } \\
& \text { not inAmerica("Carl"); } \\
& \text { inAmerica("Carl") } \leftarrow \text { sibling("Bob", "Carl"), } \\
& \text { not inEurope("Carl"). }
\end{aligned}
$$

Thus, $\operatorname{grnd}(P)$ has two answer sets, viz.

$$
\begin{aligned}
& X_{1}=\{\text { inEurope("Carl"), sibling("Bob", "Carl"), } \\
& \text { sibling("Carl", "Bob") }\} \text { and } \\
& X_{2}=\{\text { inAmerica("Carl"), sibling("Bob", "Carl"), } \\
& \text { sibling("Carl", "Bob")\}. }
\end{aligned}
$$

If we allow to forget about sibling("Carl", "Bob") in $\operatorname{grnd}(P)$, then the result of forgetting is obtained from $\operatorname{grnd}(P)$ by removing the first rule.

The above definitions of forgetting about an atom $l$ can be extended to forgetting about a set $F$ of atoms. Specifically, we can similarly define $X_{1} \subseteq_{F} X_{2}, X_{1} \sim_{F} X_{2}$, and $F$-answer sets of a HEX-program. In fact, the properties of forgetting about a single atom can be generalized to the case of forgetting about a set. Moreover, the result of forgetting about a set $F$ can be obtained by forgetting each atom one by one in $F$.

Proposition 2 Let $P$ be a consistent HEX-program and $F=\left\{l_{1}, \ldots, l_{m}\right\}$ a set of atoms. Then, forget $(P, F) \equiv$ forget $\left(\ldots\right.$ (forget $\left(\right.$ forget $\left.\left.\left.\left(P, l_{1}\right), l_{2}\right), \ldots\right), l_{m}\right)$.

Since higher-order atoms and external atoms can be treated as ordinary atoms in the process of forgetting, we can prove the above result similarly to the proof of Proposition 6 given by Eiter and Wang [4].

For HEX-programs, the notion of ordinary forgetting may not be sufficient for some applications in managing ontologies. In some cases, we need to forget a predicate. This can be easily accomplished by forgetting the set of all atoms with the same predicate.

Due to the presence of higher-order terms, we may need also to forget some other atoms when we want to forget a specific atom. This is illustrated in the following example.
Example 4 Suppose we want to forget brotherOf in the following program:

$$
\begin{array}{r}
\text { subRelation }(\text { brotherOf }, \text { siblingOf }) \leftarrow ; \\
\text { brotherOf }(\text { john }, \text { al }) \leftarrow ; \\
\text { siblingOf }(\text { john, joe }) \leftarrow ; \\
\text { siblingOf }(\text { al, mick }) \leftarrow ; \\
R(X, Y) \leftarrow \text { subRelation }(P, R), P(X, Y) .
\end{array}
$$

Here, also subRelation(brotherOf, siblingOf) should be forgotten.

For the above discussion, it is natural to define the following variant of forgetting, which is more intuitive for most applications.

Definition 3 Let $P$ be a HEX-program and $l$ an atom in $P$. Denote by $\sup (l)$ the set of all atoms in $P$ that contain the predicate name of $l$. Then the result of enforced forgetting about $l$ in $P$, written $\operatorname{Forget}(P, l)$, is defined as forget $(P, \sup (l))$.

In Example 4, Forget $(P$, brother $O f)$, given by

$$
\begin{aligned}
\text { forget }( & P,\{\text { brotherOf }(j o h n, \text { al }), \\
& \text { subRelation }(\text { brotherOf }, \text { siblingOf })\}),
\end{aligned}
$$

is the following program:

$$
\begin{aligned}
& \{\text { siblingOf }(\text { john }, \text { al }) \leftarrow ; \text { siblingOf }(\text { john }, \text { joe }) \leftarrow ; \\
& \text { siblingOf }(\text { al, mick }) \leftarrow\} .
\end{aligned}
$$

\subsection{Forgetting for Non-Ground Positive Programs}

As we will see in Section 4, the logic program $P_{L}$ translated from an OWL/RDF ontology is non-ground in general and thus forgetting as defined by Eiter and Wang [4] cannot be directly applied here. However, since $P_{L}$ has a special form and, in particular, has no negation as failure, we are able to lift the notion of forgetting for ground programs to this kind of non-ground programs.

To this end, we first need to define weak unfolding for logic programs.

Let $r: a \leftarrow b, B$ and $r^{\prime}: b^{\prime} \leftarrow B^{\prime}$ be normal rules, where $a, b, b^{\prime}$ are atoms, and $B, B^{\prime}$ are conjunctions of literals. Note that no higher-order atoms occur here. When necessary, we can rename the variables of $r^{\prime}$ such that $r$ and $r^{\prime}$ have no common variables. If the head $b^{\prime}$ of $r^{\prime}$ and $b$ have a most general unifier (mgu) $\theta$, then the rule $\left(a \leftarrow B, B^{\prime}\right) \theta$ is called a resolvent of $r$ with $r^{\prime}$.

Weak Unfolding. A logic program $P^{\prime}$ is obtained from $P$ by weak unfolding iff there are two rules $r$ and $r^{\prime}$ in $P$ such that $r^{\prime \prime}$ is a resolvent of $r$ with $r^{\prime}$ and $P^{\prime}=$ $P \cup\left\{r^{\prime \prime}\right\}$. 
Algorithm 2 (Computing forgetting for non-ground positive logic programs)

Input: Positive logic program $P$ and a predicate $R$. Output: Program forget $(P, R)$ as the result of forgetting $R$ from $P$.

Method:

1. Fully apply weak unfolding on $P$.

2. Remove all rules containing $R$.

3. Output the resulting program as forget $(P, R)$.

\section{Figure 3. Computing the result of forgetting for non-ground positive programs.}

For a positive logic program the result of forgetting can be easily obtained by Algorithm 2 depicted in Figure 3.

The following result shows that this lifting algorithm for forgetting is sound with respect to semantic forgetting for ground programs.

Theorem 1 Let $P$ be a non-ground positive program and $R$ a predicate in $P$.

For any extensional database $E$ (i.e., a set of facts), we have forget $(P, R) \cup E \equiv$ forget $(\operatorname{grnd}(P \cup E)$, const $(R))$, where const $(R)=\{R(a) \mid$ a is a constant in $P \cup E\}$.

Algorithm 2 may be refined by applying Step 1 only to a subset of the rules and facts $P$ which is relevant to $R$, while the rest of the program remains untouched. In this way, the cost of computing forgetting can be reduced radically.

For $P$ and $R$ in Algorithm 2, let $Q$ initially be the set of all rules in which $R$ appears. Then, add each rule $r$ from $P$ to $Q$ such that head $(r)$ appears in $Q$, and repeat this process until no new rules can be added. Let the resulting program be denoted by $Q_{P, R}$. Intuitively, $\bar{Q}$ consists of rules that are irrelevant to $R$. For forgetting in a positive program, it is done by a series of unfolding and then removing some rules relevant to $R$. So, rules in $\bar{Q}$ are essentially unchanged during the process of forgetting.

Theorem 2 Let $P$ be a non-ground positive program $P$ and let $R$ be a predicate in $P$. For any extensional database $E$, it holds that forget $(P, R) \cup E \equiv \operatorname{forget}\left(Q_{P, R}, R\right) \cup \bar{Q} \cup E$, where $\bar{Q}=P \backslash Q_{P, R}$.

It should be noted that although the process of forgetting for non-ground programs is realized by the removal of certain rules, it has a semantic justification as Theorem 2 shows.

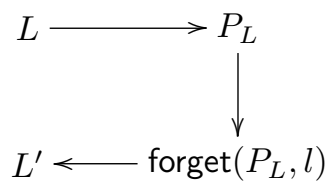

Figure 4. Forgetting in ontologies via a logic program.

\section{Forgetting in OWL/RDF-Ontologies}

To apply forgetting purely to an ontology expressed in OWL or RDFS, we reuse the techniques defined for forgetting in logic programs. Figure 4 shows the general principle of this approach. First, an ontology $L$ is translated into a rule representation $P_{L}$, taking the specific ontology semantics into account. Then, for any atom $l$ in $P_{L}$, we can compute forget $\left(P_{L}, l\right)$. Finally, we translate the result back into an ontology.

The translation of description-logic axioms into a logic program is shown in Table 1. This translation covers most of the expressiveness of OWL Lite and corresponds to the translation given by Grosof et al. [11], mapping some subset of a description logic to positive equality-free datalog programs. Note that some description-logic constructs have no direct representation in logic-programming rules, such as cardinality constraints. Also, existential and universal quantification is restricted to the left-hand side resp. righthand side of a subclass axiom. In general, a transformation from a set of rules back to ontology statements requires the rules in forget $\left(P_{L}, l\right)$ to be in a form according to Table 1.

Example 5 Consider again the ontology $L$ in Figure 2. The translation of $L$ into a logic program according to $P_{L}$ is depicted in Figure 5. Suppose we do not want to keep the concepts worksWith, then we can use Theorem 1 to simplify the process of forgetting.

Take $Q$ as a subprogram of $P_{L}$ :

$$
\begin{aligned}
& \text { sameProject("Bob", "Dennis") } \leftarrow \text {; } \\
& \text { worksWith }(X, Y) \leftarrow \text { worksWith }(Y, X) \text {; } \\
& \text { worksWith }(X, Z) \leftarrow \text { worksWith }(X, Y) \text {, } \\
& \text { works With }(Y, Z) \text {; } \\
& \text { knows }(X, Y) \leftarrow \text { worksWith }(X, Y) \text {; } \\
& \text { worksWith }(X, Y) \leftarrow \text { sameProject }(X, Y) \text {. }
\end{aligned}
$$

We can apply Algorithm 2 on the $\operatorname{logic}$ program $Q$ by forgetting works With. First, fully apply weak unfolding on $Q$ and obtain $Q^{\prime}$ :

$$
\begin{aligned}
& \text { sameProject ("Bob", "Dennis") } \leftarrow ; \\
& \text { worksWith }(X, Y) \leftarrow \text { worksWith }(Y, X) \text {; }
\end{aligned}
$$




\begin{tabular}{|l|c|rc|}
\hline Statement & DL syntax & Rule representation \\
\hline subClassOf & $D \sqsubseteq C$ & $C(X)$ & $\leftarrow D(X)$. \\
subPropertyOf & $P \sqsubseteq Q$ & $Q(X, Y)$ & $\leftarrow P(X, Y)$. \\
domain & $T \sqsubseteq \forall P^{-} . C$ & $C(X)$ & $\leftarrow P(X, Y)$. \\
range & $T \sqsubseteq \forall P . C$ & $C(Y)$ & $\leftarrow P(X, Y)$. \\
class-instance & $a: C$ & $C(a)$ & $\leftarrow$. \\
property-instance & $\langle a, b\rangle: P$ & $P(a, b)$ & $\leftarrow$. \\
class-equivalence & $D \equiv C$ & $D(X)$ & $\leftarrow C(X) ;$ \\
& & $C(X)$ & $\leftarrow D(X)$. \\
property-equivalence & $P \equiv Q$ & $P(X, Y)$ & $\leftarrow Q(X, Y) ;$ \\
& & $Q(X, Y)$ & $\leftarrow P(X, Y)$. \\
inverseOf & $P \equiv Q^{-}$ & $P(X, Y)$ & $\leftarrow Q(Y, X) ;$ \\
& & $Q(X, Y)$ & $\leftarrow P(Y, X)$. \\
transitiveProperty & $P^{+} \sqsubseteq P$ & $P(X, Y)$ & $\leftarrow P(X, Z), P(Z, Y)$. \\
\hline
\end{tabular}

\begin{tabular}{|l|c|cc|}
\hline Constructor & DL syntax & Rule representation \\
\hline conjunction & $C_{1} \sqcap C_{2} \sqsubseteq D$ & $D(X)$ & $\leftarrow C_{1}(X), C_{2}(X)$. \\
& $C \sqsubseteq D_{1} \sqcap D_{2}$ & $D_{1}(X)$ & $\leftarrow C(X) ;$ \\
& & $D_{2}(X)$ & $\leftarrow C(X)$. \\
disjunction & $C_{1} \sqcup C_{2} \sqsubseteq D$ & $\leftarrow C_{1}(X) ;$ \\
& & $D(X)$ & $\leftarrow C_{2}(X)$. \\
existential restriction & $\exists P . C \sqsubseteq D$ & $D(X)$ & $\leftarrow P(X, Y), C(Y)$. \\
universal restriction & $D \sqsubseteq \forall P . C$ & $C(Y)$ & $\leftarrow P(X, Y), D(X)$. \\
\hline
\end{tabular}

Table 1. Mapping of ontology statements and class constructors to rules.

$$
\begin{aligned}
& \text { worksWith }(X, Z) \leftarrow \text { worksWith }(X, Y) \text {, } \\
& \text { works With }(Y, Z) \text {; } \\
& \text { knows }(X, Y) \leftarrow \text { works With }(X, Y) \text {; } \\
& \text { works With }(X, Y) \leftarrow \operatorname{sameProject}(X, Y) \text {; } \\
& \text { knows }(X, Y) \leftarrow \text { sameProject }(X, Y) \text {; } \\
& \text { works With ("Bob", "Dennis") } \leftarrow \text {; } \\
& \text { knows("Bob", "Dennis") } \leftarrow \text {. }
\end{aligned}
$$

Thus, the result of forgetting about works With is the program forget $(Q$, works With $) \cup \bar{Q}$, where $\bar{Q}=P_{L} \backslash Q$ and forget $(Q$, works With $)$ is as follows:

$$
\begin{aligned}
& \{\text { sameProject ("Bob", "Dennis") } \leftarrow ; \\
& \text { knows }(X, Y) \leftarrow \text { sameProject }(X, Y) ; \\
& \text { knows("Bob", "Dennis") } \leftarrow\} .
\end{aligned}
$$

Translating this fragment back into the original description logic results in the following statements:

$$
\begin{aligned}
& \text { sameProject ("Bob", "Dennis"); } \\
& \text { sameProject } \sqsubseteq \text { knows; knows("Bob", "Dennis"). }
\end{aligned}
$$

The property works With does not occur in the modified description-logic knowledge base any more, while the subproperty relation between sameProject and knows is preserved.

Combining the approaches to forgetting of Sections 3 and 4 , we are now able to forget any set of ordinary atoms, higher-order atoms, whole external atoms, and parts of external atoms in a HEX-program.

\section{Related Work and Concluding Remarks}

The notion of forgetting for HEX-programs introduced in this paper generalizes a respective notion for ordinary logic programs defined in previous work [4]. Forgetting for HEX-programs provides a means to handle forgetting at the user-view level, since HEX-programs are tailored to access sources like OWL/RDF ontologies at the extensional level through external atoms, but does not go back to changes in these sources, as is done in the view-update problem of databases, for instance. However, such ontologies have been cast to a class of logic programs which constitute a small fragment of HEX-programs, and thus semantic forgetting for OWL/RDF may be facilitated through this mapping, as we have shown. Our work therefore provides a uniform basis for a framework for extracting ontology segments from a custom ontology, which is exploited at the user level. This approach is in an active area of semantic integration in ontologies (see [17] for a survey). However, the emphasis of our work is on conflict resolving in semantic integration of ontologies rather than on ontology mapping.

Forgetting for OWL/RDF ontologies can be used for various tasks in ontology management including the following:

- Ontology segmentation: This approach is to obtain segments from a custom ontology, thus having the same purpose as forgetting. Seidenberg and Rector [20] present a series of strategies for extracting ontology segments. However, it lacks a general semantic justification. 
